\title{
Transitions and probes in turbulent helium
}

\author{
Virginie Emsellem, ${ }^{1}$ Leo P. Kadanoff, ${ }^{2,}{ }^{*}$ Detlef Lohse, ${ }^{2,3, \dagger}$ Patrick Tabeling, ${ }^{1}$ and Z. Jane Wang ${ }^{2, \dagger}$ \\ ${ }^{1}$ Laboratoire de Physique Statistique, Ecole Normale Supérieure, 75231 Paris cedex 05, France \\ ${ }^{2}$ The James Franck Institute, The University of Chicago, Chicago, Illinois 60637 \\ ${ }^{3}$ Fachbereich Physik der Universität Marburg, Renthof 6, 35032 Marburg, Germany
}

(Received 19 April 1996)

\begin{abstract}
Previous analysis of a helium turbulence experiment by Zocchi et al. and Tabeling et al. [Phys. Rev. E 50, 3693 (1994); 53, 1613 (1996)] shows a transition at the Taylor Reynolds number $\operatorname{Re}_{\lambda} \approx 700$. Here correlation function data are analyzed which gives further evidence for this transition. It is seen in both the power spectrum and in structure function measurements. Two possible explanations may be offered for this observed transition: that it is intrinsic to the turbulence flow in this closed box experiment or that it is an effect of a change in the flow around the anemometer. We particularly examine a pair of "probe effects." The first is a thermal boundary layer which does exist about the probe and does limit the probe response, particularly at high frequencies. Arguments based on simulations of the response and upon observations of dissipation suggest that this effect is only crucial beyond $\mathrm{Re}_{\lambda} \approx 2000$. The second effect is produced by vortex shedding behind the probe. This has been seen to produce a large modification in some of the power spectra for large $\operatorname{Re}_{\lambda}$. It might also complicate the interpretation of the experimental results for higher values of the Reynolds number. However, there seems to be a remaining range of data for $\operatorname{Re}_{\lambda}<1300$ uncomplicated by these effects, and which are thus suggestive of an intrinsic transition. [S1063-651X(97)04602-3]
\end{abstract}

PACS number(s): 47.27. $-\mathrm{i}, 47.80 .+\mathrm{v}$

\section{INTRODUCTION}

Recent velocity measurements in highly turbulent helium gas flow by Tabeling et al. [1-6] reveal a transition in the turbulent behavior. The results of Refs. [1-5] show that beyond some crossover Taylor Reynolds number $\operatorname{Re}_{\lambda} \approx 700$ the flatness of the velocity derivatives ceases to increase. Such a transitional behavior has never been reported in open flows $[7,8]$, while earlier results on closed flows, also in helium [9-11], have also revealed the existence of a transition at large Reynolds numbers. In our discussions we would distinguish between two classes of experiments: (1) A group of experiments on thermally driven turbulence in helium, conducted by Libchaber and co-workers (see Refs. [9-11]). We shall collectively describe these experiments as TTH, and (2) the other group of experiments include studies of sheardriven turbulence in helium (STH). This set includes Refs. [1-6] which have been done by Tabeling and co-workers. For the STH experiments, it has been argued that the crossover signals the instability of vortex tubes (worms) [5]; one may speculate also whether it signals the onset of Kolmogorov 1941 (K41) turbulence [13].

This paper is devoted to a further analysis of this crossover, including two main questions: (1) Can the transition be seen in the further analysis of correlation functions? (2) Can it possibly be understood as some kind of probe effect?

To discuss the former issue we analyze structure functions $D_{n}(r)=\left\langle[u(x+r)-u(x)]^{n}\right\rangle$ of order $\mathrm{n}=2,4,6$ and the energy spectrum. We try to superpose structure functions and spectra for different Reynolds numbers Re. Indeed, the su-

\footnotetext{
*Electronic address: leo@control.uchicago.edu

${ }^{\dagger}$ Electronic address: lohse@cs.uchicago.edu

‡Electronic address: jw@control.uchicago.edu
}

perpositions show that there are different forms of the curves above and below the transition, but within each group the forms of the curves are roughly the same. The shifts necessary to superpose such curves give a measurement of characteristic length or frequency scales in each measurement. These scales and their dependence upon the Reynolds number will be analyzed in this paper because they are important to give insight into the physical origin of the transition.

The central point of this paper is to examine the possibility that the apparent transition might be a reflection of probe defects. We study two different specific probe effects in some detail.

There are two likely sources of difficulties in the measurement, one being that the probe might have insufficient time resolution, the other being that it might be too large. To study the first possibility, we follow the analysis of Grossmann and Lohse [14], who showed the possibility of the loss of probe sensitivity in temperature measurements for Rayleigh-Bénard flow $[15,9]$. This probe effect occurs as a result of delays and averaging related to thermal diffusion through the partially stagnant gas about the probe. In the STH experiments considered in the present paper, the probe is a hot wire anemometer, which works by heating the gas around it. Once again, the probe measures temperature changes and can possibly have poor temporal response. This is the first probe effect we analyze in this paper. The second kind of effect we consider involves what happens when the probe size and the dissipative scale becomes comparable. One effect which can arise is vortex shedding behind the probe. In "ordinary" hot wire anemometry (i.e., cylindrical probes working in air), the operating conditions are such that even at large (overall) Reynolds numbers, there is no vortex shedding behind the sensors [16]. In the STH experiments, the Reynolds number based upon probe size is large enough so that it is reasonable to ask about vortex shedding. This 
issue has been partially addressed in the past $[2,4]$; the general conclusion is that if the vortex shedding frequency is outside the turbulent spectrum, no big perturbation is expected. In the opposite case, the vortex shedding mode may couple with the turbulent fluctuations, and perturb the measurement. Our analysis compares these scales and discusses the implication of the results.

The paper is organized in the following way. In Sec. II, we show evidence for a change in behavior at high Reynolds numbers. Section III is devoted to looking at probe effects which might partially explain the observed change in behavior. In Sec. IV, additional evidence is drawn from the measured Reynolds number dependence of characteristic scales. In Sec. V we demonstrate by a simple estimate that inertial subrange (ISR) quantities, such as scaling corrections or probability distribution functions (PDFs) of velocity differences in that range, are hardly effected by the transition. Section VI is devoted to conclusions.

\section{EVIDENCE FOR TRANSITION}

\section{A. The flatness}

The velocity measurements of the STH experiments are done in low temperature helium gas, following the idea of the TTH experiments $[17,15,9]$ which were built upon the experiments of Threifall [18]. The flow is driven by two counterrotating disks of radius $R$. Two different cells are used: Cell 1 with $R=3.2 \mathrm{~cm} \mathrm{[3,1,2]} \mathrm{and} \mathrm{cell} 2$ with $R=10$ $\mathrm{cm}[1,2]$. The velocity anemometer ("probe") of size $d=7$ $\mu \mathrm{m}$ is placed far enough from the boundary layers. The Reynolds number is defined as $\operatorname{Re}=\Omega R^{2} / \nu$. The angular velocity $\Omega$ of the disks, which is about $1-10 \mathrm{~Hz}$, remains about the same for all measurements, whereas Re is varied by changing the helium pressure and thus the viscosity $\nu$.

The transition was first observed [2] in the properties of the flatness $F$ and the skewness of the velocity derivative. In this paper, we focus upon the flatness since the transitional phenomenon was less visible in the skewness. The flatness is defined to be

$$
F=\lim _{r \rightarrow 0} \frac{D_{4}(r)}{\left[D_{2}(r)\right]^{2}} .
$$

Figure 1 shows a series of flatness measurements for the small and large cells, plotted as a function of $\mathrm{Re}_{\lambda}$. This Reynolds number is experimentally defined in terms of the rms fluctuations in the velocity [1]. Figure 1 incorporates unpublished data recently obtained with the smaller cell. A rough estimate for the experimental uncertainty in the flatness is $\pm 15 \%$. At a lower Reynolds number, the flatness $F$ increases with increasing $\operatorname{Re}_{\lambda}$. Then, at a transition value of the Reynolds number, it seems to reach a peak and then level off at a higher $\operatorname{Re}_{\lambda}$. The peak flatness is located at $\operatorname{Re}_{\lambda}$ comprised between 550 and 750 for the small and large cells (see Fig. 1 and also Fig. 5 of [2]). These peak values are identified as the $\operatorname{Re}_{\lambda}$ number for some kind of crossover or transition.

The nature of the transition reflects itself in the $\operatorname{Re}_{\lambda}$ dependence of characteristic length scales. A possible choice is the length scale of the transition from the viscous subrange (VSR) to the inertial subrange (ISR) in the velocity structure

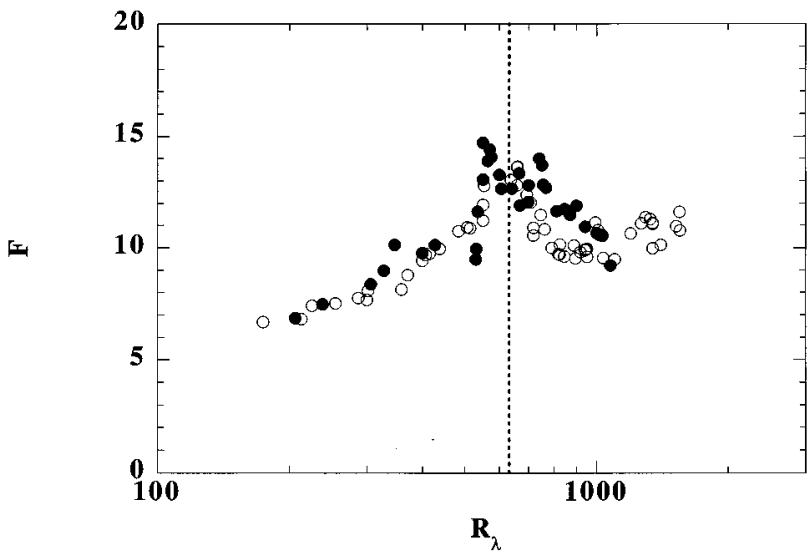

FIG. 1. The flatness of the velocity derivatives is plotted against $\operatorname{Re}_{\lambda}$. Results for the small cell are given as the black points and for the large one as the empty circles. This same convention is used in the later figures. Each point shown here is the average of three experiments performed at the same $\operatorname{Re}_{\lambda}$, in the same cell, with the same probe.

function $D_{3}(r)$. In Ref. [1] the length scale $l_{\text {inf }}$ was defined as the value for which the ratio $-D_{3}(r) / r$ is equal to $70 \%$ of its maximum value and we will adopt this definition here. The classical expectation is that a length like this will decrease as $\mathrm{Re}^{-3 / 4}$. The experimental result shows the expected behavior below a transitional Reynolds number but appears to saturate at higher Reynolds numbers. (See Fig. 5 in Ref. [1].) It was also found (see [1]) that the scaling range of apparent ISR behavior seems to saturate and may even shrink beyond the transition Reynolds number.

\section{B. Dissipation spectra and structure functions}

The previous work mostly focused on the scalings of the viscous dissipation. Here we seek further evidence of the transition and its nature. We first analyze the energy dissipation spectra $I(k)=k^{2} E(k)$ as a function of the Reynolds number. [Here, $E(k)$ is the usual power spectrum, obtained as a function of frequency and then translated into a dependence upon wave vectors through its mean velocity following the Taylor hypothesis.] We find that the curves for the dissipation spectra fall into two groups: There is one shape which holds for all curves below the transition and another shape which governs the curves above the transition.

The spectra are calculated over 16 million points, corresponding to 100-1000 large eddy turnover times for each graph. The data is then divided into about $2^{9}$ sequencies of $2^{15}$ points. The power spectra are computed for each sequence and averaged over different segments. A Hanning window is used in computing the spectra [19]. To collapse the spectra, we determine the peak of the dissipation $I_{p}$ and its corresponding frequency $k_{p}$ following the approach of Ref. [20]. We then rescale the wave number and the dissipation spectrum by $k_{p}$, and $I_{p}$, respectively. The rescaled spectra are then plotted in Fig. 2. Figure 2(a) shows the collapse for different Reynolds numbers below the transition, whereas Fig. 2(b) for those above the transition. Figure 2(c) shows a comparison of the shapes for the two scaling curves. The solid line corresponds to a general fitting form taken from $[20,21]$ 

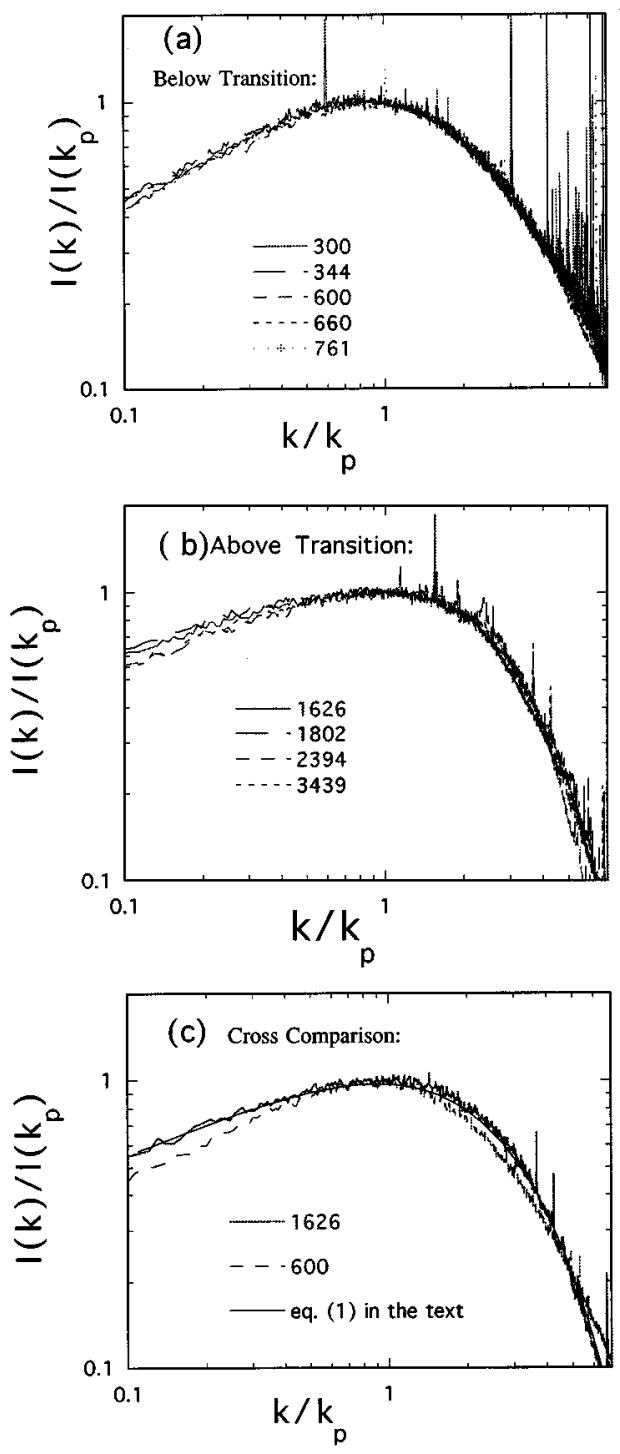

FIG. 2. Collapse of dissipation spectra. Parts (a) and (b), respectively, show overlays of different dissipation spectra for $\operatorname{Re}_{\lambda}$ below and above the transitional value. Part (c) shows the comparison of two spectra, one from above and one from below.

$$
I(k)=I\left(k_{p}\right)\left[\left(\frac{k}{k_{p}}\right)^{-5 / 3}+\alpha\left(\frac{k}{k_{p}}\right)^{-\beta}\right]\left(\frac{k}{k_{p}}\right)^{2} e^{-\mu k / k_{p}},
$$

where the fitting parameters are $\alpha=0.7, \beta=-1$, and $\mu$ is determined to make the peak occur at $k / k_{p}=1$. In this fit, the second summand reflects the bottleneck energy pileup at the borderline between inertial subrange and viscous subrange [22]. Note the good agreement between the fitting formula and the data holds above the transition, but not below. Figure 2 then shows a situation in which the dissipation spectra changes at the transitional Reynolds number.

The relative shifts in the logarithmic scales define unambiguously the relative length and energy scale in the system. In Sec. IV, we shall study the Reynolds number dependence of the length scales in comparison with different theories of what might cause the apparent transition.

To further test the existence of two groups of scaling functions, we examine different structure functions $D_{n}(r)$ in the same spirit. First we look at $D_{2}$. Since $D_{2}$ and the power spectra contain the same information, the results on $D_{2}$ should provide us a double check on the previous finding. To collapse the different curves of $D_{2}$ versus $r$, we use the same method as applied to the dissipation spectra. We find a characteristic length for each plot by finding the point of the log-log plot at which the slope has a predetermined constant value. To probe the VSR-ISR transition range, we choose the constant slope to be 1.5 , i.e., a value in between the VSR slope 2 and the ISR slope of approximately $2 / 3$. The method corresponds to finding the maximum of $D_{2}(r) / r^{1.5}$. We call this scale $r_{\text {max }}^{(2)}$ and define $D_{2, \max }=D_{2}\left[r_{\text {max }}^{(2)}\right]$. In Fig. 3 we plot $D_{2}(r) / r^{1.5}$ vs $r$ for various $\operatorname{Re}_{\lambda}$, normalized so that the maximum always is at $(1,1)$. Once again, we find the data separate into two groups: one for $\operatorname{Re}_{\lambda}<800$, and another for $\operatorname{Re}_{\lambda}>1500$. The relative shifts in horizontal and vertical directions define the scale in length and velocity. We return to the length scale in Sec. IV.

We apply the same method to study $D_{4}(r)$ and $D_{6}(r)$. We again collapse them by finding specific values of the slope in the log-log plot. In these cases, the chosen slopes are, respectively, 1.5 and 2.5. For the fourth order structure function (Fig. 4), we see clear separation of two groups. Again, the separation occurs between $\operatorname{Re}_{\lambda}=800$ and $\operatorname{Re}_{\lambda}=1500$. However, Fig. 5 for the sixth order structure functions does not show such separations. The failure of seeing two groups in $D_{6}$ somewhat weakens the argument for a simple transition.

Thus we have shown some additional evidence for the existence of a transition in the STH experiment. The structure functions $D_{2}(r), D_{4}(r)$, and the spectrum indicate a simple transition whereas higher order structure functions suggest a broadened transition.

It is clear that there is some kind of change or transition centered at $\operatorname{Re}_{\lambda}$ of about 700. How does one explain the observed transition? Can it possibly be a probe effect? We turn to those issues in Sec. III.

\section{PROBE EFFECTS}

In this section, we shall obtain a variety of order of magnitude estimates. To make these estimates, we will have to compare a characteristic dissipative frequency in the system to the characteristic inverse times produced by the probe itself. We estimate the dissipative frequency as $\omega_{d}=U /(10 \eta)$ in agreement with the spectral measurements of Zocchi et al. [1]. This frequency is then the inverse of the time it takes for a disturbance of size $10 \eta$ to move past the probe. Here, we use the classically expected [23] relation between the Kolmogorov length $\eta$ and the dissipation rate $\epsilon$. The definition of the length is $\eta=\nu^{3 / 4} / \epsilon^{1 / 4}$, with $\eta$ having the approximate value

$$
\frac{\eta}{R}=30 \mathrm{Re}^{-3 / 4}
$$

In our last steps of analysis we shall express our results in terms of the Taylor-Reynolds number which according to the measurements of Ref. [1] is connected to the Reynolds number by 


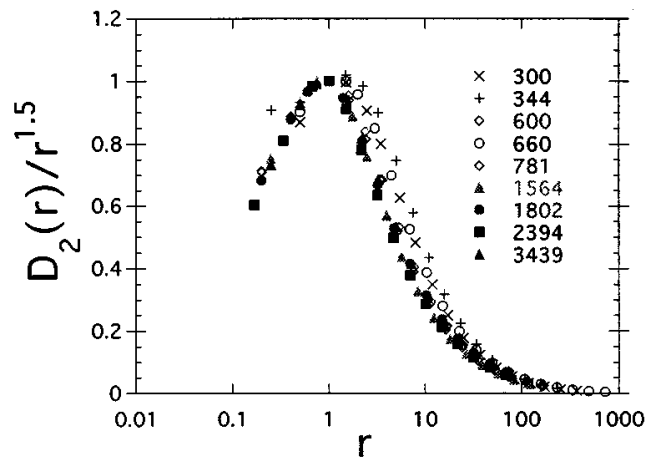

FIG. 3. The curves $D_{2}(r) / r^{1.5}$ vs $r$ group into two groups, one for Reynolds numbers below the transition, one for Reynolds numbers above the transition. We have shifted the curves so that the maximum is at $(1,1)$. As in Fig. 2, this figure also contrasts the relatively unvarying behavior above and below the transition with the somewhat larger change which occurs across the transition. In this and in the next two figures, we use open symbols to indicate the flows below transition, and solids above the transition.

$$
\operatorname{Re}_{\lambda}=1.57 \mathrm{Re}^{1 / 2} .
$$

From Eq. (2) we obtain

$$
\omega_{d}=\frac{U}{10 \eta}=\frac{\nu}{R^{2}} \frac{1}{300} \operatorname{Re}^{7 / 4}
$$

Here and below, we do our estimates by setting the Prandtl number, defined as the ratio of viscosity and the thermal diffusivity, equal to unity.

\section{A. The thermal boundary layer}

The experimental results [1] on the crossover length scale $l_{\text {inf }}$ in the third order structure function resemble those deduced from the temperature measurements in the highly turbulent Rayleigh-Bénard flow [9]: Beyond a critical Rayleigh number $\mathrm{Ra} \approx 10^{11}$, the scaling range of the temperature

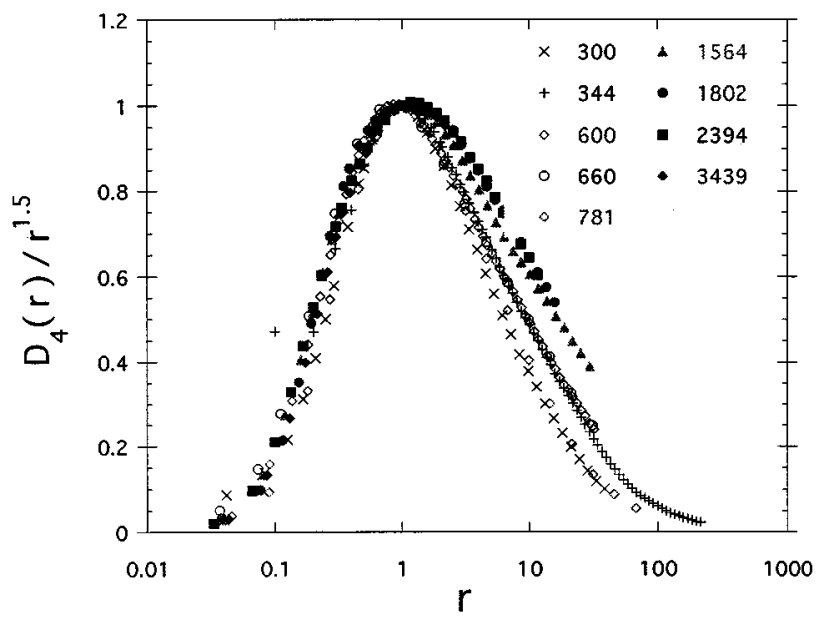

FIG. 4. $D_{4}(r) / r^{1.5}$ vs $r$; again, both axes are normalized so that the maximum is at $(1,1)$. As in Fig. 3 this figure contrasts the behavior within the region above and below the transition with the change which occurs across the transition.

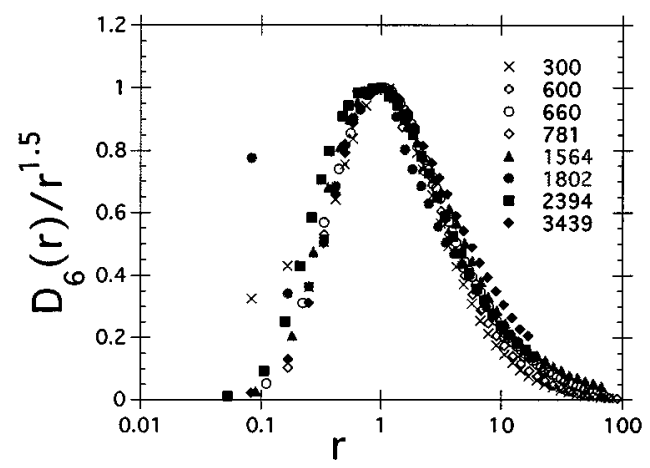

FIG. 5. $D_{6}(r) / r^{2.5}$ vs $r$, normalized as in the two previous figures. In contrast to Figs. 3 and 4, a separation of the curves into two groups is not obvious.

power spectrum becomes smaller for increasing $\mathrm{Ra}$ and the measured dissipative power shows a weaker Ra increase as below the transition. Grossmann and Lohse [14] suggested that this apparent transition might really be an effect caused by the probe used to measure temperatures. The thickness $\delta$ of the boundary layer around the probe sets a diffusive time scale

$$
\omega_{\delta}^{-1}=\delta^{2} / \kappa,
$$

where $\kappa$ is the heat diffusivity. Beyond $\omega_{\delta}$, the measured spectral strength will be reduced. If $\omega_{\delta}$ is smaller than the UV spectral cutoff $\omega_{d}$, this will affect the UV side of the inertial range between $\omega_{\delta}$ and $\omega_{d}$. This explanation of the observed high Rayleigh number data as a probe effect is still unproven, but it has certainly never been ruled out.

One might wonder whether a similar effect would affect the results of the STH experiments. Note that the simplest estimate for the value of $\operatorname{Re}_{\lambda}$ at the transition of the TTH experiment is $\left(10^{11}\right)^{1 / 4} \approx 700$. By some accident (?!) this is the same number as that observed in the STH experiments.

Why is heat diffusion relevant for the velocity measurements? The anemometer is heated by an electrical current. The faster the fluid is passing by the probe, the more power is needed to keep the probe at some constant temperature, which is larger than the temperature of the surroundings. The probe gauge curve power vs velocity is given by King's law and is experimentally known [3].

What velocities does the probe measure? It cannot be the velocity directly at the probe, as there will be a viscous boundary layer of thickness $\delta$ around it in which the velocity is very small. The heat generated in the probe has to diffusively penetrate this layer. Thus the probe measures the velocity of the helium which is a distance $\delta$ away from the probe. The length scale $\delta$ again sets the diffusive time scale described by Eq. (5).

Our problem is to now estimate the important value(s) of $\delta$ and then to see whether the $\omega_{\delta}$ thereby generated provides an important cutoff on the responsiveness of the probe.

This has been carried out in two different ways. One of us (V.E.) has done numerical simulations of the flow past the probe, assuming a laminar time-independent flow [24]. The calculation must be done numerically since the Reynolds numbers of the probe

$$
\operatorname{Re}_{\text {probe }}=\frac{U d}{\nu}=\operatorname{Re}_{R}
$$


is of the order of 40 at the observed transition point. Here, $d=7 \mu \mathrm{m}$ is the size of the probe. A steady state is reached in which the flow is constant and in which there is a constant flow of heat away from the probe. The calculated flow is then perturbed with a sudden upstream rise in temperature. The probe responds and the important result is that the probe response time is shorter than any time resolved in the experiment. The conclusion is that one can assume that the thermal response of the probe is perfect.

The next question is: How large is the viscous boundary layer $\delta$ ? There are, in fact, two answers. If the probe Reynolds number is of order 10 to 100 , then there are regions with a thin viscous boundary layer in which the flow comes very close to the cylinder. According to the Blasius theory this boundary layer thickness should be

$$
\delta \propto d / \sqrt{\operatorname{Re}_{\text {probe }}} .
$$

The flow behind the probe produces a much larger region of stagnant fluid at rest, with a thickness comparable to the cell size. Thus we have also

$$
\delta \propto d .
$$

The frequency produced by these lengths via Eq. (5) will only matter if they are smaller than or of the order of the Kolmogorov cutoff of Eq. (4). Since these frequencies differ by a factor of the probe Reynolds number they are quite different. The conditions they generate are different also. If the length $d$ matters, then the frequency cutoff will bother us whenever the Reynolds number Re obeys

$$
\operatorname{Re}>\left[300\left(\frac{R}{d}\right)^{2}\right]^{4 / 7} .
$$

Conversely if the size of the Blasius boundary layer matters, we will get the less stringent condition

$$
\operatorname{Re}>(300 R / d)^{4 / 3}
$$

for the Reynolds number at which the thermal boundary layer of the probe becomes important.

These answers are quite different. If the important distance is $d$, we get from Eq. (9) an estimate of the critical Taylor-Reynolds number, $\operatorname{Re}_{\lambda}$, as $\approx 1000$ for the small cell and $\approx 2000$ for the large cell. The experiment measures a crossover $\operatorname{Re}_{\lambda}$ of order 700 . These results are perilously close.

On the other hand, if it is appropriate to use the Blasius length then Eq. (10) gives a much larger critical Reynolds number, in fact, a critical value of $\operatorname{Re}_{\lambda}$ which is greater than $10^{4}$ and which cannot be realized in the experiment.

So which length should we use? To see the answer notice that the experiment measures the heat flow out of the probe. This heat flow is much larger in regions in which the boundary layer is thinner. Thus, for larger $\operatorname{Re}_{\text {probe }}$ the important regions are the ones which have the Blasius effect and are thinned by a factor of $\left(\operatorname{Re}_{\text {probe }}\right)^{-1 / 2}$. The simulation [24] fully supports this point of view. Note also that this line of argument does not apply to the TTH experiment $[15,9]$ and its analysis [14]. In the TTH experiment, the probe measures the average temperature in its environment. Regions of the thick boundary layer are as important as thin ones. Therefore, relatively sluggish regions can effect the outcome. In contrast, in the STH experiments, only the most responsive regions matter.

\section{B. The dissipation rate test}

One can construct a direct test of the probe responsiveness. The energy dissipation rate-denoted by $\epsilon$ - can be measured in two different ways [1]. One involves a measurement in the inertial subrange and determines a quantity which we call $\epsilon_{I S R}$. The other is a dissipative measurement and determines $\epsilon_{V S R}$. Both quantities can be expressed in dimensionless form by writing $c$ as the ratio of $\epsilon$ to $U^{3} / R$ where $U=\Omega R$ is the large scale velocity. In this way one finds the two different (Reynolds number dependent [25]) ratios

$$
c_{\epsilon, I S R}=\epsilon_{I S R} \frac{R}{U^{3}} \quad \text { and } \quad c_{\epsilon, V S R}=\epsilon_{V S R} \frac{R}{U^{3}} .
$$

One can find $\epsilon_{I S R}$ by using the fact that the third order structure function $D^{(3)}(r)$ obeys the Kolmogorov structure equation [23]

$$
D^{(3)}(r)=-4 \epsilon_{I S R} r / 5 .
$$

When Tabeling's group carries out their ISR measurement their results agree with the expected spatial scaling and thus enable them to construct $c_{\epsilon, I S R}$. In addition, the energy dissipation rate $\epsilon$ is measured by a method which uses the viscous subrange, $\epsilon_{V S R}$, namely, by determining $\epsilon$ from the spectrum $E(k)$ as

$$
\epsilon_{V S R}=15 \nu \int_{0}^{\infty} d k k^{2} E(k) .
$$

Here, full isotropization has been assumed. Strictly speaking, $\epsilon_{V S R}$ is only based on $\left\langle\left(\partial_{1} u_{1}\right)^{2}\right\rangle$ which is (via Taylor's hypothesis [26]) the only experimentally accessible contribution to the strain tensor $\partial_{i} u_{j}$. This determination of $\epsilon$ then gives the other dimensionless constant, $c_{\epsilon, V S R}$. In Fig. 6 we show the ratio $c_{\epsilon, I S R} / c_{\epsilon, V S R}$. If the probe response was cut off at high frequencies, one would expect $c_{\epsilon, V S R}$ to be substantially smaller than $c_{\epsilon, I S R}$, i.e., the ratio to be larger than 1. The data shows no support for that hypothesis. So we must conclude that, within experimental error (which is rather substantial), the high frequency response of the probe is satisfactory.

\section{Vortex shedding behind the probe}

In this section, we discuss the effect of finite probe size on spatial resolution. We focus particularly upon the effects of vortex shedding from the probe.

Zocchi et al. [1] have observed a series of peaks at the high end of the power spectra. The exact origin of these peaks is unknown. Possible sources are vortex shedding, vibrations of the probe and its support. In more recent experiments, the fiber has been strained at a tensile strength ten times larger than before and the peaks have mostly disappeared. This indicates that some of the observed peaks were 


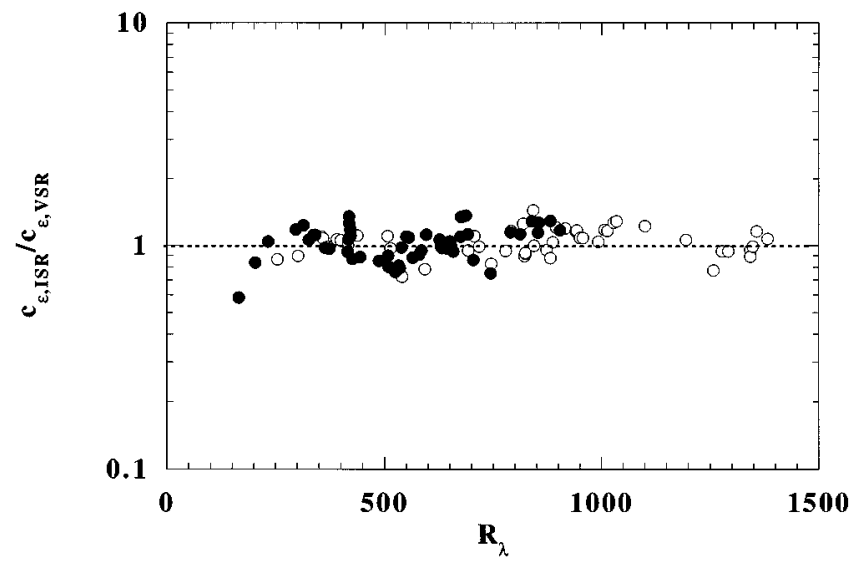

FIG. 6. Ratio of $c_{\epsilon, I S R}$ and $c_{\epsilon, V S R}$ vs $\operatorname{Re}_{\lambda}$. Each point is the average of three experiments performed at the same $\operatorname{Re}_{\lambda}$, in the same cell, with the same probe. Notice that the two measurements agree within experimental error. Earlier measurements published in [1] showed an apparent discrepancy between $c_{\epsilon, I S R}$ and $c_{\epsilon, V S R}$ with the dissipative range quantity being smaller than the integral range one. Those measurements lead to the assumption that probe effects degraded the data at high frequencies $[27,14]$. The plot here shows the latest measurements of Tabeling and co-workers performed at smaller fluctuation rates (around 20\%, to be compared to $35 \%$ for [1]), and do not reveal any discrepancy between $\epsilon_{I S R}$ and $\epsilon_{V S R}$.

a vibration of the fiber. Here, we study the vortex shedding to estimate where it will occur and the effect it is likely to have.

The frequencies of vortex shedding can be estimated as

$$
f_{v}=\mathrm{St} \frac{U_{0}}{d}
$$

where St is the Strouhal number, which is typically 0.2 [28]; $U_{0}$ is the velocity of an ambient fluid, and $d$ is the diameter of the obstacle. We focus upon the shedding from the probe, which has a diameter $d \sim 7 \mu \mathrm{m}$.

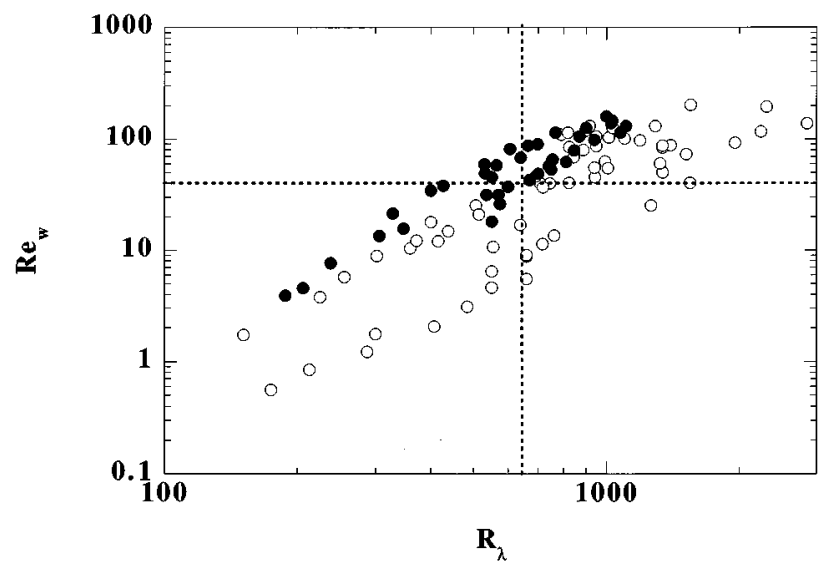

FIG. 7. The various probe Reynolds numbers $\operatorname{Re}_{w}$ we achieve in the experiment for the two cells. Black points refer to the small cell, open circles to the large one. The scatter is partly due to the fact that several fluctuation rates are considered in the compilation. The line shows the critical value, $\operatorname{Re}_{w} \sim 40$, for the onset of vortex shedding.

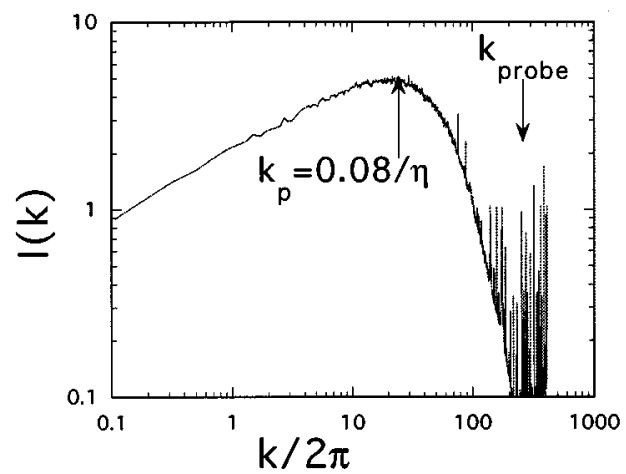

FIG. 8. The spectra for $\operatorname{Re}_{\lambda}=1626$. The arrows mark the positions of the estimated Kolmogorov wave number, and the one of the vortex shedding.

Two conditions must be satisfied for the vortex shedding to be important. First it must be present. Vortex shedding appears when the probe Reynolds number is above $\sim 40$ [28]. In Fig. 7 we plot the probe Reynolds number $\operatorname{Re}_{w}$ as a function of $\operatorname{Re}_{\lambda}$. The horizontal dashed line corresponds to the known [28] onset value 40. Its crossing with the experimental data gives a reading of transitional value in $\mathrm{Re}_{\lambda}$, which is between 600 and 800 for small cell, and 600-1100 for large cell. These ranges are close to the observed transition in flatness. Next, the shedding frequency must be in an observable range of frequency. With the typical values $U_{0} \sim 1 \mathrm{~m} / \mathrm{s}$ in Eq. (14), we estimate the vortex shedding frequency to be about $15 \mathrm{kHz}$, which is in the observable range of frequency. A typical spectrum is shown in Fig. 8.

Does vortex shedding have an important effect upon the flatness? Since the flatness is measured as a scale on the order of typically $5 \eta$, we expect the vortex shedding to begin to affect the flatness when the vortex shedding frequency [Eq. (14)] is comparable with the Kolmogorov frequency [Eq. 4)]. Under Taylor's hypothesis, this is equivalent to comparing the shedding wavelengthes $l_{v}$ and the Kolmogorov length $\eta$. The shedding wavelength is defined by

$$
l_{v}=\frac{U_{0}}{2 \pi f_{v}} \sim 1.2 d
$$

and thus essentially equals the probe size $d$. In Fig. 9 we plot

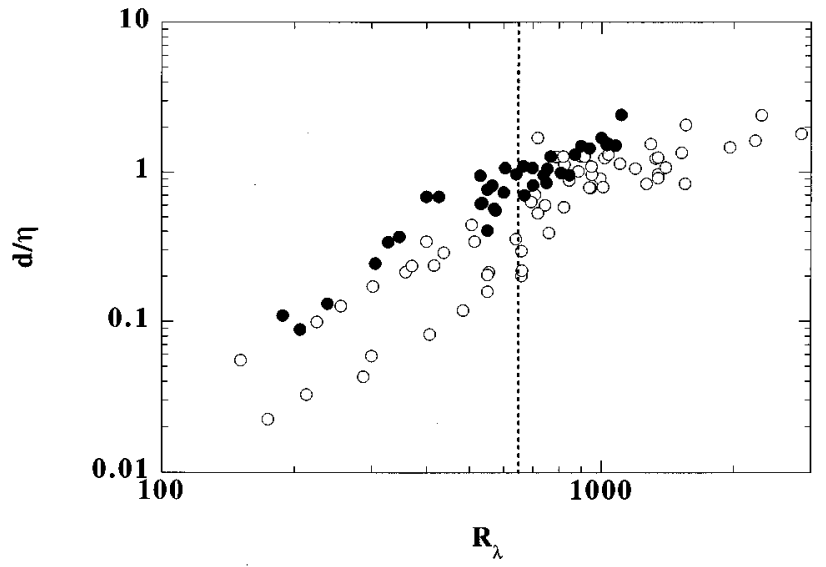

FIG. 9. The probe size $d$, measured in multiples of $\eta$, as a function of $\operatorname{Re}_{\lambda}$. The symbols are as in the preceding figure. 
the probe size $d$ (in multiples of $\eta$ ) as a function of $\operatorname{Re}_{\lambda}$. Once again, we find that the line $1.2 d$ crosses the data around the same range of transitional value in $\operatorname{Re}_{\lambda}$ as seen in the flatness. Thus the vortex shedding shows promise of explaining the position of the transition.

Next we want to know how the vortex shedding affects the measurements of the flatness. Let us decompose the velocity signal $U(t)$ as

$$
U(t)=V(t)+A \sin \left(\omega_{v} t\right),
$$

where $V(t)$ denotes the velocity in the absence of shedding and the other term might reflect, for example, a shaking of the probe with angular frequency $\omega_{v}$. We know that the intrinsic signal $V(t)$ gives a very large flatness, of order 10 . On the other hand, a sinusoidal signal, like the term in $A$, will give a much smaller flatness. Thus we should expect that such an additional term would tend to reduce the flatness, as seen in the experiments. A careful calculation bears out this point [29].

However, Figs. 7 and 9 show some puzzling features. Note the probe Reynolds number is defined as in Eq. (6). For a fixed geometry we expect $\operatorname{Re}_{\lambda}$ to be a fixed constant times $\mathrm{Re}^{1 / 2}$. Thus all the black dots (corresponding to the small cell) in Fig. 8 should fall on one straight line with slope $1 / 2$ and all the white dots should fall on another, lower by an amount corresponding to the log of the ratio of the cell sizes, $\log _{10} 3$. So why do the large cell data fall onto two lines? Figures 1 and 7 collect data measured in two distinct cells: one is $3 \mathrm{~cm}$ in radius, and the other one is $10 \mathrm{~cm}$ in radius. They cover four series of experiments performed over a year; there is no simple relation between $\operatorname{Re}_{\lambda}$ and $\mathrm{Re}$, because, for the same cell size, we may have substantially different integral scales due to the fact that the rims do not have the same size from one series to the other. Moreover, we have worked with different velocity fluctuation rates. All this explains why, for a given cell size, we do not have a simple relation between $\operatorname{Re}_{\lambda}$ and $\mathrm{Re}$. However, if we fit the means of the two lines we get a reasonable value of the separation between the curves. This separation realizes our theoretical expectation that the two intersection points should have $\operatorname{Re}_{\lambda}$ values which differ by a factor of the square root of the size ratio or roughly 1.7 .

On the other hand, according to Fig. 1 there is no obvious difference in the transition point between the larger cell and the smaller. This lack of difference would be expected if the transition were an intrinsic effect; it is not expected if it results from the probe size via having $\operatorname{Re}_{w}$ with a constant value at the transition. Thus, Fig. 8 seems to explain the position of the transition as a probe effect, but cannot satisfy us on the question of the size dependence. We do note that the uncertainties in the measurements are large, thus a possible size dependence may be overlooked.

Now we return to the question on the size of the probe relative to $\eta$. The shedding wavelength [Eq. (15)] is independent of the Reynolds number. On the other hand, the maximum dissipative frequency gives a scale $10 \eta$ which decreases with Reynolds number as $\mathrm{Re}^{-3 / 4}$. If $10 \eta$ becomes comparable or smaller than $d$, the measurements of the dissipative quantities, such as the length measurements discussed in Sec. IV, will be influenced by the finite probe size.
Figure 9 shows that, in fact, the probe size is considerably smaller than $10 \eta$. Consequently, the vortex shedding may give such high frequencies that it is unlikely to affect the measured length while it may affect the flatness. (The reader will recognize that we are getting onto dangerous ground, since we are trying to distinguish between $\eta$ and $10 \eta$ in an order of magnitude argument.)

What does one expect to see in the length measurements if the probe effect is relevant? As we have already seen, the vortex sheddings introduce to the system an external length scale which is independent of the Reynolds number. This length scale becomes observable if the measurements of interest are on the order of the probe size. This suggests a saturation of the VSR-ISR crossover scale $l_{\text {inf }}$ above the transition, which is consistent with Fig. 5 in Ref. [1]. Similarly, it predicts that the length scales divided by the Kolmogorov scale increase with Reynolds number as $\operatorname{Re}_{\lambda}^{3 / 2}$. The comparisons will be done in Sec. IV.

Finally we might remark that the whole problem of this paper is in distinguishing intrinsic effects from probe effects. We tried to do this by separating the effects of $\operatorname{Re}_{\lambda}$ from that of the probe Reynolds number. We did not entirely succeed. In a future experiment, one might want to hold $\operatorname{Re}_{\lambda}$ fixed at a few values near the transition while varying the probe Reynolds number. It might just be possible to do this since the average velocity and the root mean square fluctuations in velocity can be varied in a somewhat independent fashion. This technique might serve to distinguish probe effects from intrinsic effects. Until we can make this distinction in a sharper fashion, we shall remain somewhat dissatisfied. For now, we see an interesting and plausible transition whose existence has been suggested but not definitely proven.

\section{CHARACTERISTIC LENGTHS: MEASUREMENTS VS PREDICTIONS}

In this section, we discuss the characteristic lengths displayed by the turbulence data. Below the transition, we expect that the Kolmogorov dissipation length provide the characteristic scale for all short-distance phenomena. Beyond the transition, we expect that another length might enter the problem. In both the vortex shedding approach and the thermal boundary layer approach, this other length turns out to be of the order of the size of the probe. This result is obtained for the thermal boundary layer by substituting Eq. (7) into Eq. (5) and multiplying by the integral scale velocity. The result is a length which is essentially the probe size. In this section, we measure all lengths in units of the dissipation length. Therefore, we expect to see a constant value for this ratio below the transition. If the probe dominates the behavior by either of the mechanisms discussed here, we expect the ratio of characteristic length to dissipation length to increase as $\mathrm{Re}_{\lambda}^{3 / 2}$.

Our first method defines the dissipation lengths by identifying the maximum energy dissipation scale $k_{p}$ as we did to collapse the dissipation spectra. Figure 10 shows $k_{p}$ normalized by $k_{\eta}$ as a function of Reynolds number. The overall behavior can be fitted by a constant as shown by the horizontal line. If the probe affects the scales when $\operatorname{Re}_{\lambda}>700$ we might expect the ratio to slope upward as shown. Either a 


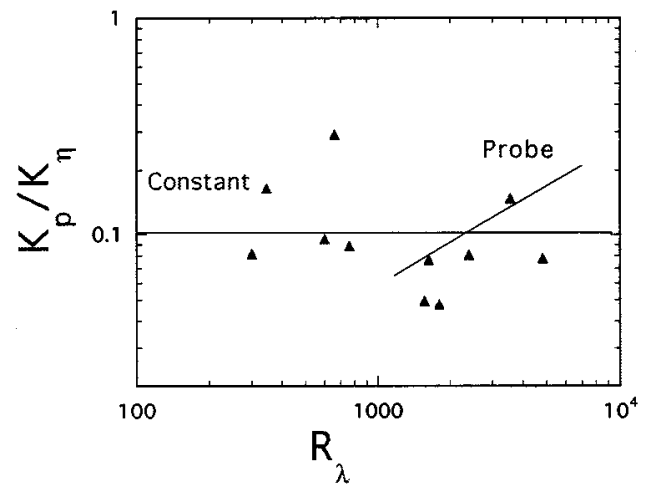

FIG. 10. The position $k_{p}$ of the maximum of the dissipation spectrum $I(k)$ defined in Eq. (1) as a function of $\operatorname{Re}_{\lambda}$.

constant or an upward slope is equally well supported by these data.

Similarly, we can analyze the $\operatorname{Re}_{\lambda}$ dependences of the lengths scales obtained by identifying the relative shifts used to collapse $D_{2}(r), D_{4}(r)$, and $D_{6}(r)$ as discussed in Sec. II. These lengthes normalized by $\eta v^{v s} \operatorname{Re}_{\lambda}$ are shown in Fig. 11 . Again, a horizontal line is draw across them, and compared with the prediction of the two probe scenarios. These data do not support the notion of an upward slope. Hence, they suggest that the scales of order $30 \eta$ are not affected by probes unlike the flatness measurements, which correspond to scales of $5 \eta$.

Next, we define the crossover length to be the length $r_{\min }$ corresponding to the minima of the second log-log derivative of $D_{2}$. Here, $r_{\min }$ should capture the crossover from a $r^{2}$ scaling behavior at small $r$ to a $r^{0.7}$ scaling in the inertial range. The Reynolds number dependence of $r_{\min }$ is shown in Fig. 12 in comparison with the probe prediction. No evidence for a probe effect is seen. This is not too surprising because here we are dealing with lengths of the order of $20 \eta$, whereas in flatness measurements are done in much smaller length scales. The Reynolds number dependence of all these length ratios seems to be consistent with both a constant and the probe predictions within the scatter of data.

In contrast, the related VSR-ISR crossover length scale $l_{\text {inf }}$ calculated in Ref. [1] did show a saturation beyond the

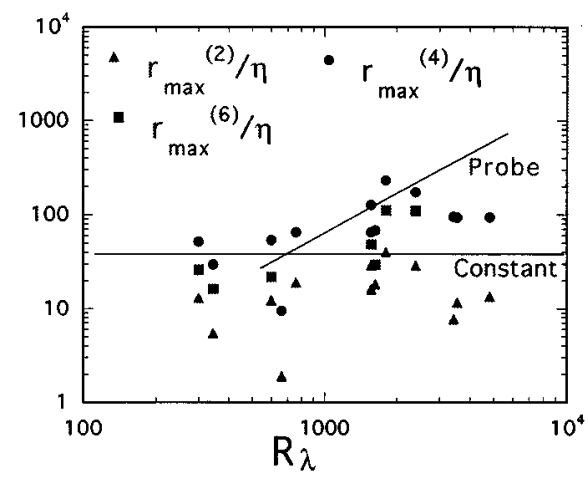

FIG. 11. The positions $r_{\max }^{(2)}, r_{\max }^{(4)}$, and $r_{\max }^{(6)}$ of the maxima of $D_{2}(r) / r^{1.5}, D_{4}(r) / r^{1.5}$, and $D_{6}(r) / r^{2.5}$, respectively. These lengths are the relative shifts used to collapse $D_{2}(r), D_{4}(r)$, and $D_{6}(r)$ for the various $\operatorname{Re}_{\lambda}$. They are measured in multiples of $\eta$.

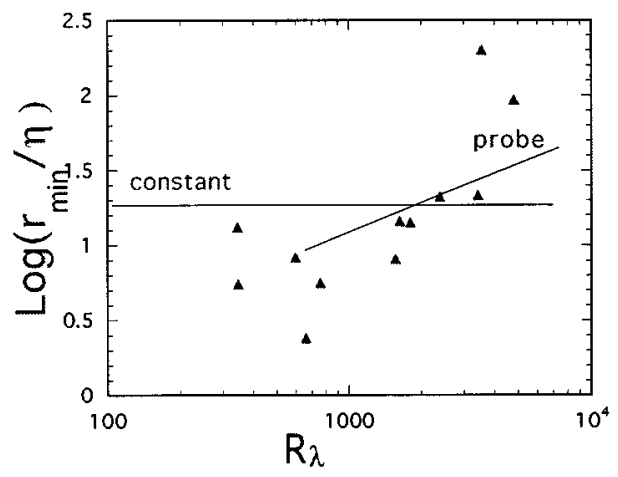

FIG. 12. Position $r_{\min }$ (in multiples of $\eta$ ) of the mimimum of $d^{2} \ln D_{2}(r) / d(\ln r)^{2}$ vs $\operatorname{Re}_{\lambda}$.

transitional Reynolds number. This is suggestive of the existence of an external scale in the system. The plot of $l_{\text {inf }}$ is Fig. 5 in Ref. [1].

\section{CAN THE TRANSITION BE SEEN IN THE ISR?}

The transition in the flatness of $\partial_{1} u_{1}$ at $\operatorname{Re}_{\lambda_{c}} \sim 700$ is rather pronounced, as seen from Fig. 1. How does this transition towards K41 turbulence in a VSR quantity reflect in pure ISR quantities? In this section we will see that for experimentally reachable Reynolds numbers the transition can hardly be expected to be visible in ISR quantities as velocity structure function exponents and PDFs of ISR quantities. This finding is in agreement with the measurements of Tabeling et al. $[1,2,4]$. Thus the ISR results of the STH experiments do not contradict the existence of the transition towards K41 turbulence.

For our estimate here it is sufficient to sketch the flatness $F(r)=\left\langle v_{r}^{4}\right\rangle /\left\langle v_{r}^{2}\right\rangle^{2}$ as follows [30]:

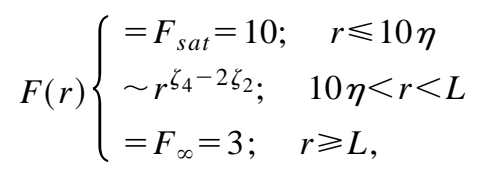

$L$ is the integral length scale. From [1] we have $L=4 \mathrm{~cm}$ $=0.4 R$ for the large cell, independent of $\operatorname{Re}_{\lambda}$. From the sketch (17) we immediately obtain the $\operatorname{Re}_{\lambda}$ dependence of the scaling corrections to K41 turbulence,

$$
\delta\left(\operatorname{Re}_{\lambda}\right)=\left|\zeta_{4}\left(\operatorname{Re}_{\lambda}\right)-2 \zeta_{2}\left(\operatorname{Re}_{\lambda}\right)\right|=\frac{\ln \left(F_{s a t} / F_{\infty}\right)}{\ln \left[L / 10 \eta\left(\operatorname{Re}_{\lambda}\right)\right]}
$$

The ratio $L / 10 \eta$ scales like

$$
\frac{L}{10 \eta}=c \operatorname{Re}_{\lambda}^{3 / 2}
$$

Rather than taking $c$ from Eqs. (1) and (2) of Sec. II we adopt it to the experimental value of $\delta$ for the Reynolds number $\operatorname{Re}_{\lambda}=650$ of the transition. From [4] we have $\zeta_{4}=1.25$ and $\zeta_{2}=0.70$. Thus $\delta=0.15$ and $c=0.185$. The $\operatorname{Re}_{\lambda}$ dependence of the scaling correction $\delta\left(\operatorname{Re}_{\lambda}\right)$ is very weak and approaches its K41 value only logarithmically, $\delta\left(\operatorname{Re}_{\lambda}\right) \propto 1 / \operatorname{lnRe} e_{\lambda}$, suggesting that $1 / \ln \operatorname{Re}_{\lambda}$ rather than 
TABLE I. Reynolds number dependence of ISR characteristics for various Reynolds numbers beyond the transition. Only weak dependence is detected.

\begin{tabular}{lccc}
\hline \hline $\operatorname{Re}_{\lambda}$ & $\delta\left(\operatorname{Re}_{\lambda}\right)$ & $F\left(490 \mu \mathrm{m}, \mathrm{Re}_{\lambda}\right)$ & $\beta\left(\mathrm{Re}_{\lambda}\right)$ \\
\hline 650 & 0.150 & 5.82 & 1.02 \\
1000 & 0.139 & 5.54 & 1.06 \\
1500 & 0.130 & 5.32 & 1.09 \\
3000 & 0.117 & 5.03 & 1.14 \\
5000 & 0.109 & 4.85 & 1.18 \\
\hline \hline
\end{tabular}

$1 / \operatorname{Re}_{\lambda}$ is the small parameter in turbulence. Some numbers for still experimentally reachable Reynolds numbers are given in Table I. This weak decrease is in agreement with the slight experimental decrease of the scaling correction $\delta \zeta_{n}$ as shown in Fig. 8 of [4].

Now we focus on the PDF of $v_{r}$. In Fig. 2 of Ref. [2] no detectable dependence of the PDF for $v_{r}$ for fixed scale $r=490 \mu \mathrm{m}$ on the Reynolds number was noticed. With our above sketch (17) of $F(r)$ we readily calculate

$$
F\left(\frac{r}{L}, \operatorname{Re}_{\lambda}\right)=3\left(\frac{r}{L}\right)^{-\delta\left(\operatorname{Re}_{\lambda}\right)} .
$$

The Reynolds number dependence of $F\left(r / L, \operatorname{Re}_{\lambda}\right)$ for fixed $r / L=490 \mu \mathrm{m} / 4 \mathrm{~cm}=0.012$ again is very weak, Table $\mathrm{I}$.

Finally, we give as a characteristic of the PDF $\mathcal{F}$ also the stretching exponent $\beta$ in a parametrization

$$
\mathcal{F}\left(v_{r}\right) \propto \exp \left(-\left|\frac{v_{r}}{v_{r}^{0}}\right| \beta\right)
$$

which is well known to fit the tails of experimental PDFs. $\beta=2$ means Gaussian PDF. The flatness and the stretching exponents are connected by [30]

$$
F=\frac{\Gamma(1 / \beta) \Gamma(5 / \beta)}{[\Gamma(3 / \beta)]^{2}} .
$$

The stretching exponent $\beta$ again only very weakly depends on the Reynolds number, Table I. They must be directly compared with Fig. 2 of Ref. [2] where also hardly any dependence is seen.

To summarize this section: The transition towards K41 turbulence, seen in the STH experiments, cannot be expected to be pronouncedly seen in pure ISR quantities as PDFs for fixed $r$ or scaling exponents $\zeta_{n}$. To look for a similar transition in other geometries one should thus focus on VSR quantities as, e.g., the (hyper)flatnesses.

\section{CONCLUSIONS}

The STH experiments show a transition in behavior for $\operatorname{Re}_{\lambda} \approx 700$. This transition can be seen in the flatness and indeed in measurements of individual moments like the power spectrum and low order velocity moments. However, one might worry that the apparent transition was caused by the finite size or finite frequency resolution of the probe. We have analyzed herein two effects which may affect the measurement performed in the STH experiments: (a) the thermal response of the probe, which imposes a limitation on the temporal resolution and (b) the vortex shedding behind the probe, which distorts the spatial resolution of the probe. What should we conclude?

The two probe scenarios discussed in this paper both sound plausible. We cannot prove that they are the direct cause of the transition, and in fact, we find partial evidence against each. In the end, we conclude that the measurements up to $\operatorname{Re}_{\lambda} \sim 1300$ are probably not perturbed by the two effects which are discussed in the present paper.

We suspect the thermal boundary layer effect to be relevant at higher Reynolds number $\sim 2000$, but not in the region where the transitional behavior is observed. The key point here is the presence of a boundary layer which enhances the thermal exchanges between the fiber and the surrounding fluid. This is confirmed by numerical analysis [24], which showed that the thermal frequency response is outside the turbulent spectrum in a comfortable range of Reynolds number around the transition. This is further checked by the observation that the dissipation rate seems to be correctly measured in the same range of Reynolds number; therefore the thermal response of the probe seems to be satisfactory for the measurement of dissipative quantities.

Although the vortex shedding scenario gives the right transition value in $\operatorname{Re}_{\lambda}$ and produces the observed decrease of flatness with $\operatorname{Re}_{\lambda}$, it also predicts a size dependence of the transitional Reynolds number. However, the latter does not appear to be supported by the experiment. We also remark that a set of improved experiments show less of the anomalous peaks in the spectra, and yet the transition persists.

Perhaps neither the vortex shedding nor the thermal layer is in itself the right explanation. However, the transition does occur when the probe Reynolds number is high (of order 40) and when the dissipation length is of the same order of magnitude as the probe size. There are likely to be many other possible effects, not explored here, which only depend on two essential ingredients: (1) the finite size of the probe and (2) the injection of energy in the small scale comparable to the size of the probe. Thus another effect of finite probe size or response might intervene and produce a false signal of a transition. On the other hand, it is entirely possible that the transition observed is real and has nothing to do with probe effects.

To fully understand the nature of the transition, we will require further experiments with closed flows. They are likely to involve helium and probes similar to those employed here. Clearly, it would be very desirable if a major piece of the next experiment were devoted to understanding further the behavior of the probes, and of the flow in their neighborhood, and how this flow evolved with the Reynolds number. To fully realize the potential of the experimental method, we need further development of the technique for using probes like these. Perhaps one can probe the velocity and temperature field around large probes with tiny ones to further understand the probe effects.

\section{ACKNOWLEDGMENTS}

Very helpful exchanges with F. Belin, L. Biferale, B. Castaing, J. Eggers, S. Grossmann, K. R. Sreenivasan, G. Stolovitzky, H. Willaime, and G. Zocchi are acknowledged. 
We also thank Kadanoff and co-workers and Tabeling and co-workers for their hospitality during visits from one institution to another. This work has been supported by DOE Contract No. DE-FG02-92ER25119, by the MRSEC Pro- gram of the National Science Foundation under Grant No. DMR-9400379, by the DFG through its SFB185, by CNRS, Universités Paris 6 and Paris 7, Ecole Normale Superieure, and the NATO grant under Contract No. CRG-950245.
[1] G. Zocchi, P. Tabeling, J. Maurer, and H. Willaime, Phys. Rev. E 50, 3693 (1994).

[2] P. Tabeling, G. Zocchi, F. Berlin, J. Maurer, and H. Willaime, Phys. Rev. E 53, 1613 (1996).

[3] J. Maurer, P. Tabeling, and G. Zocchi, Europhys. Lett. 26, 31 (1994)

[4] F. Belin, P. Tabeling, and H. Willaime, Physica D 93, 52 (1996).

[5] F. Belin, P. Tabeling, and H. Willaime, J. Phys. 6, 573 (1996).

[6] F. Belin, J. Maurer, P. Tabeling, and H. Willaime, J. Phys. (France) II 6, 573 (1996).

[7] C. W. van Atta and R. A. Antonia, Phys. Fluids 23, 252 (1980).

[8] For a comprehensive recent review, see K. R. Sreenivasan and R. A. Antonia, Annu. Rev. Fluid Mech. 29, 435 (1997).

[9] I. Procaccia et al., Phys. Rev. A 44, 8091 (1991).

[10] X. Z. Wu, L. Kadanoff, A. Libchaber, and M. Sano, Phys. Rev. Lett. 64, 2140 (1990).

[11] B. Chabaud, A. Naert, J. Peinke, F. Chilla, B. Castaing, and B. Hebral, Phys. Rev. Lett. 73, 3227 (1994).

[12] J. Jimenez, A. Wray, P. Saffman, and R. Rogallo, J. Fluid Mech. 255, 65 (1993).

[13] A. N. Kolmogorov, C.R. Acad. Sci. USSR. 30, 299 (1941).

[14] S. Grossmann and D. Lohse, Phys. Lett. A 173, 58 (1993).

[15] B. Castaing et al., J. Fluid Mech. 204, 1 (1989).
[16] J. O. Hintze, Turbulence (McGraw-Hill, New York, 1975).

[17] F. Heslot, B. Castaing, and A. Libchaber, Phys. Rev. A 36, 5870 (1987).

[18] D. C. Threifall, Ph.D. thesis, University of Oxford, 1976 (unpublished); J. Fluid. Mech. 67, 1 (1975).

[19] William H. Press et al., Numerical Recipes (Cambridge University Press, Cambridge, England, 1986), p. 425.

[20] Z. S. She and E. Jackson, Phys. Fluids A 5, 1526 (1993).

[21] S. Grossmann and D. Lohse, Phys. Fluids 6, 611 (1994); Phys. Rev. E 50, 2784 (1994).

[22] G. Falkovich, Phys. Fluids 6, 1411 (1994); D. Lohse and A. Müller-Groeling, Phys. Rev. Lett. 74, 1747 (1995).

[23] A. S. Monin and A. M. Yaglom, Statistical Fluid Mechanics (MIT Press, Cambridge, MA, 1975).

[24] V. Emsellem, C. R. Acad. Sci. Paris, Serie IIb 322, 11 (1996).

[25] D. Lohse, Phys. Rev. Lett. 73, 3223 (1994); S. Grossmann, Phys. Rev. E 51, 6275 (1995); K. R. Sreenivasan and G. Stolovitzky, ibid. 52, 3242 (1995).

[26] G. I. Taylor, Proc. R. Soc. London Ser. A 164, 476 (1938).

[27] K. R. Sreenivasan (unpublished).

[28] D. J. Tritton, Physical Fluid Dynamics, 2nd ed. (Clarendon Press, Oxford, 1988), p. 28.

[29] J. Wang (unpublished).

[30] S. Grossmann and D. Lohse, Europhys. Lett. 27, 347 (1993). 